\title{
High-enthalpy Flow Investigations by UV Laser-induced Fluorescence
}

\author{
Hao Yan $^{1,2}$, Shaohua Zhang ${ }^{1}$, Fangyi Wang ${ }^{1,2}$, and Xilong $\mathbf{Y u}^{1,2}$ \\ ${ }^{1}$ Institute of Mechanics, Chinese Academy of Sciences, China \\ ${ }^{2}$ School of Engineering Science, University of Chinese Academy of Sciences, China
}

\begin{abstract}
A dual-dye-laser Laser-induced Fluorescence (LIF) system is established for flow field investigations in $\mathrm{JF}-10$, a $\mathrm{H}_{2} / \mathrm{O}_{2}$-detonation-driven shock tunnel. The shock tunnel generates $400 \mathrm{~mm}$-wide hypersonic flow with total enthalpy of $16.1 \mathrm{MJ} / \mathrm{kg}$ and static pressure at only105Pa, where LIF measurement is extremely challenging. In this LIF system, the laser sheet of a conventional planar LIF is rotated 90 degrees along its propagation direction, so that the fluorescence signal collected by the camera concentrates on a sharp line. The $\mathrm{S} / \mathrm{N}$ ratio is increased, thus single-shot measurements are achievable.

The rotational temperature of the flow measured by two-line thermometry (TLT) with simultaneous excitation of two $\gamma(0,0)$ transitions of $\mathrm{NO}$ at distinct rotational levels. Compared with single-dye-laser set-up, the current system is less affected by the fluctuation of the flow field from each facility operation, thus capable of reflect the dramatic temperature change across the shock layer. With precise control of the proper of the laser, and through evaluation of the error of the data, the temperature measurement and numerical simulation in the flow is consistent. Meanwhile, the velocity of the flow is measured by molecular tagging velocimetry (MTV) with a long-life fluorescence excitation, and the result agrees with the expected value. The UV LIF technique is proven useful in flow diagnostics in high-enthalpy regime.
\end{abstract}

\section{INTRODUCTION}

For the development of hypersonic flights and re-entry vehicles, ground based testing facilities such as wind tunnels are very essential as reproducing hypersonic flows at relatively low cost. Therefore, the investigation on these facilities is always the focus of aerodynamics, especially the data acquired in these facilities by varieties of diagnoses, which is the touchstone for validating of numerical models with real gas effects or Rarefied gas effects. Among those diagnostics, the Planar laserinduced fluorescence (PLIF) technique got a lot of attention duo to its particular advantages, e.g., non-intrusive, instantaneous response, high spatially resolved, and so on. It makes the PLIF very applicable and significant for hypersonic flows that are rather non-uniform and complicated because of turbulence-chemistry interactions, while laser absorption spectrometry only provides averaged results along the optical path. At the same time, studies in the past few decades have shown that the PLIF technique is a very utility way for flow visualization and quantitative measurements, like the diagnosis of intermediate species, velocity, temperature or other parameters for various flows [1-7].

Nevertheless, up until now, the quantitative measurement for high-enthalpy turbulent reacting flow with PLIF technique is still a huge challenge. because almost all fluorophores exhibit strong quenching effects in high-enthalpy flows, giving insufficient signal strength to analysis [810]. Besides, ground based testing facilities such as wind tunnels or shock tubes are usually operated by single pulse mode, as a consequence, the microstructural fluctuation of the flow field from each facility operation is also be an issue as well as the collection efficiency of the jumbo ground based testing facilities, especially for the quantitative measurement by dual rotational lines excitation PLIF technique (two-line thermometry). In the case, we established a dual-dye-laser Laser-induced Fluorescence system for flow field investigations and applied it successfully on a $\mathrm{H}_{2} / \mathrm{O}_{2}$-detonationdriven shock tunnel (model: JF-10, total enthalpy: $16.1 \mathrm{MJ} / \mathrm{kg}$, static pressure: $105 \mathrm{~Pa}$ ). With exciting the fluorescence of NO molecules by dual-dye-laser LIF apparatus, not only the temperature distributions of free-stream and the flow field around blunt body with shock layer were measured, the velocity of the flow was measured by molecular tagging velocimetry (MTV) with a long-life fluorescence excitation as well.

\section{EXPERIMENTAL SET-UP}

\subsection{JF-10 Shock Tunnel}

JF-10 is a $\mathrm{H}_{2} / \mathrm{O}_{2}$-detonation-driven shock tunnel. It has a $6.2 \mathrm{~m}$ Forward-running Detonation Cavity (FDC) driver section with inner diameter of $150 \mathrm{~mm}$, and a $12.5 \mathrm{~m}$ driven section with inner 
diameter of $100 \mathrm{~mm}$. Before a shock tunnel operation, the driver section is filled with $\mathrm{H}_{2} / \mathrm{O}_{2}$-gas mixture with ratio of $4: 1$ at $2.5 \mathrm{Mpa}$, and the driven section is filled with test gas at $10 \mathrm{kpa}$. During the shock tunnel operation, the test gas in the stagnation chamber, heated to $7890 \mathrm{~K}$ by the reflected shock, has a total enthalpy of $16.1 \mathrm{MJ} / \mathrm{kg}$. Attached to the end of the driven section is a nozzle with exit diameter of $500 \mathrm{~mm}$, where the gas is accelerated to Mach 11.7 and static pressure is reduced to $105 \mathrm{pa}$. With this setup, the concentration of NO is expected to be $4.5 \times 10^{14} \mathrm{~cm}^{-3}$ from Computational Fluid Dynamics (CFD) simulations. Previous pitot pressure measurement showed that the test flow has a uniform area of $400 \mathrm{~mm}$ in diameter.

Table 1: Parameters of JF-10 shock tunnel.

\begin{tabular}{|l|l|}
\hline Free-stream pressure (pa) & 105 \\
\hline Free-stream Mach number & 11.7 \\
\hline Total enthalpy (MJ/kg) & 16.1 \\
\hline Stagnation Chamber Temperature (K) & 7890 \\
\hline Stagnation Chamber Pressure (MPa) & 17.9 \\
\hline
\end{tabular}

\subsection{Optical Set-ups}

The main LIF apparatus, sketched in Fig. 1, includes two narrow band frequency-doubled dye laser, which are pumped by Nd:YAG lasers, two ICCDs, and other fused silica optics that redirects and shapes the laser beam. The outputs of the Nd:YAG (Model: Pro250, Spectra-Physics,) laser are the third harmonics(355nm with maximum energy of $300 \mathrm{~mJ}$ per pulse). The tunable lasers (Sirah, Model: PRSC-GA-30, Spectra-Physics) were both loaded with C450 dye and equipped with frequency conversion unit for second harmonic generation. The pulse energies were monitored by energy meter pulse by pulse.

The dye lasers were both tuned to the $\gamma(0,0)$ band of NO around $226 \mathrm{~nm}$ with a linewidth of $0.24 \mathrm{~cm}^{-1}$. The frequency double outputs of the dye laser (the single pulse energy was set around $5 \mathrm{~mJ}$, pulse width is about $10 \mathrm{~ns}$ ) were redirected by a right-angle prism at its total internal reflection surface, and then split by a 9:1 beam splitter. The major portion of the laser output was sheeted to a laser sheet with height of $50 \mathrm{~mm}$ and thick of $\sim 0.3 \mathrm{~mm}$ by lens system before induced into the test region of shock tunnel through a fused silica window. The focus of the spherical lens is behind the imaging area to avoid nonlinear effects in the area being interrogated. The thickness of the laser sheets is about $0.3 \mathrm{~mm}$ in the imaging area. The main ICCD (Princeton Instruments PM4-1024i) mounted with a $100 \mathrm{~mm}$ UV lens is placed on top of the testing section. Attached to the lens is the ZWB3 and a 315-420 nm notch filter combination that allows transmission between 250 and $315 \mathrm{~nm}$. The filters blocked the scattered excitation laser and were tested to have $40 \%$ transmittance to the fluorescence light in the NO cell.

The minor one was directed to a chamber with 3-way optical access containing diluted NO for wavelength tuning and monitoring. The accurate excited laser wavelengths were determined by maximizing the fluorescence signal of the image captured by an ICCD (Model: Andor iStar DH734-18U-03, Oxford Instruments) aiming at the NO chamber, while the in-suit overlaps of NO absorption lines and the excitation lasers were also recorded by another ICCD(Model: PI-Max4, minimum optical gate width: $2 \mathrm{~ns}$, Princeton Instruments) through a Fabry-Perot interferometer (around $266 \mathrm{~nm}$ ).

As mentioned above, the concentration of NO in JF10 shock tunnel is expected to be $4.5 \times$ $10^{14} \mathrm{~cm}^{-3}$ from Computational Fluid Dynamics (CFD) simulations. At the same time, the collection efficiency of the apparatus for fluorescence signal on the JF10 shock tunnel is unlikely to exceed $10^{-3}$ calculated from the lens aperture $(\sim 50 \mathrm{~mm})$ of ICCD and its the distance from laser sheet $(\sim 1500 \mathrm{~mm})$ with the transmittance of filters. Thus, it could be concluded that the effective concentration of NO in JF10 shock tunnel for PLIF detecting is about $10^{11} \mathrm{~cm}^{-3}$ which is far lower than the detection limit of PLIF $\left(\sim 10^{15} \mathrm{~cm}^{-3}\right.$, according to reference [13]. It means that the measurement for gas flow in JF10 shock tunnel is a huge challenge even for the laser induced fluorescence technique whose detection limit is about $10^{12}$ molecules $/ \mathrm{cm}^{3}$ according to the reference [1]. Therefore, in our experiment, the conventional horizontal laser sheet which is parallel to the image reception area of ICCD was rotated 90 degree to a vertical one perpendicular to the receiving ICCD to improve the acquired signals of NO fluorescence. The experimental results demonstrated that the signal to noise ratio of NO fluorescence in JF10 shock tunnel has been notably improved by 


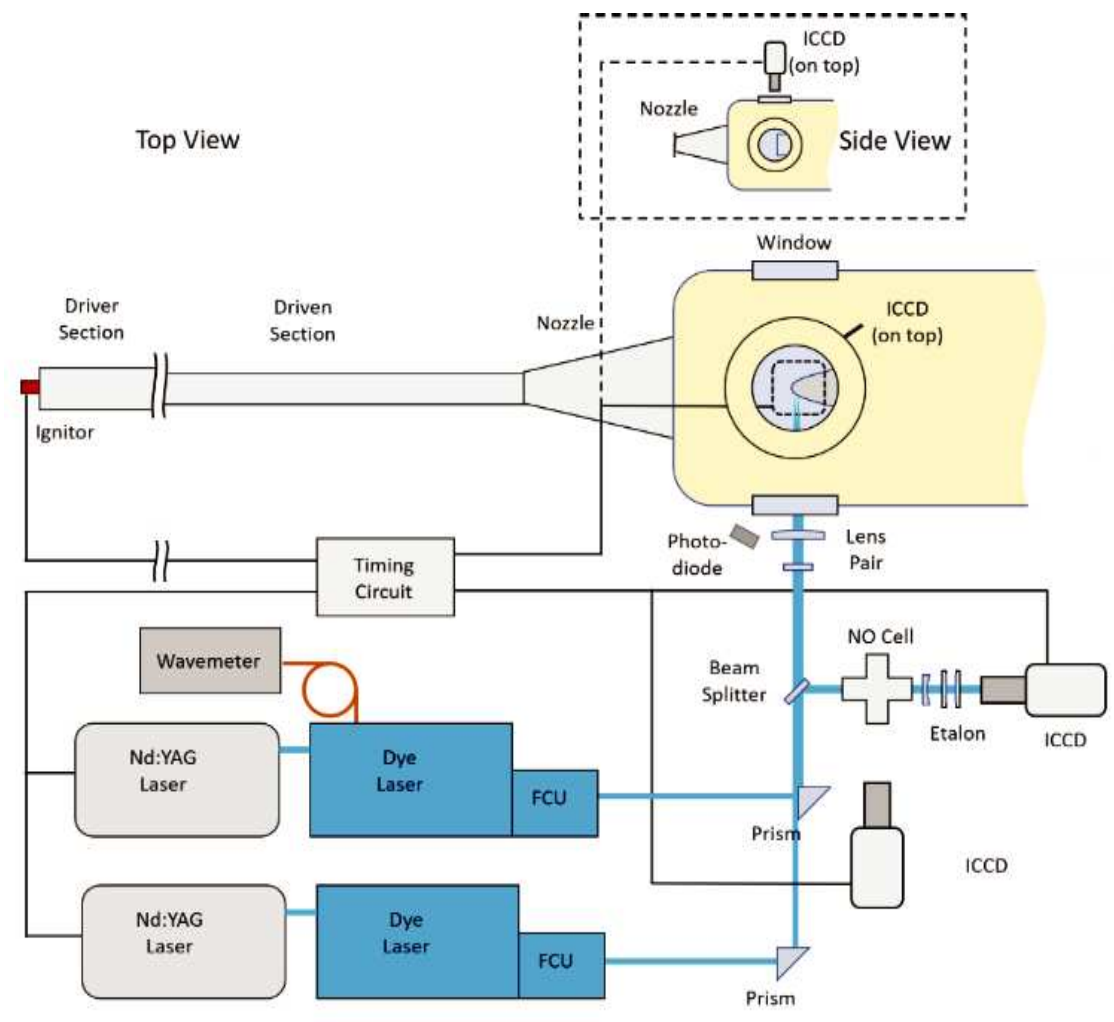

Figure 1: A schematic diagram of experimental setup and measurement.

this kind of arrangement.

\section{RESULTS}

\subsection{Two-line Thermometry on Free-stream Flow in JF-10 Shock Tunnel}

Firstly, we applied a single beam laser PLIF to the JF-10 shock tunnel for temperature measurement. A $100 \mathrm{~mm}$-wide vertical laser sheet is used for temperature measurement of free-stream flow in JF-10 shock tunnel. Fig. 2 (above) shows two LIF images taken with R21(18.5) and Q1+P21(27.5) excitations [11].

The signal strength normalized by pulse energy. The signal strength of each position in dashed lines is a summation of the signal counts over the pixels in the vertical direction on the image with background subtracted. The scattered points represent the mean value over $5 \mathrm{~mm}$ range, and its error bar represents the standard deviation of the data with the range.

According to two-line thermometry (TLT) with simultaneous excitation of two $\gamma(0,0)$ transitions of NO at distinct rotational levels, the distribution of temperature in JF-10 shock tunnel with free-stream mode could be deduced from NO-LIF images displayed in Fig. 2. As is shown in Fig. 2(below), the rotational temperature curve calculated based on these two shots is plotted in dashed line, while the scattered symbols represent the averaged value and standard deviation for all neighboring data points within $5 \mathrm{~mm}$. The mean value of the temperature is $600 \mathrm{~K}$ and the unsmoothed values has $6 \%$ standard deviation. The random error originated from the laser energy fluctuation is estimated to be $7 \%$. The result has a good agreement with TDLAS (Tunable diode laser absorption spectroscopy) measurement on the translational temperature of the free-stream in JF-10 based on the NO's absorption line shape [12].

\subsection{Two-line Thermometry on High-enthalpy Reacting flow around Blunt Body}

As JF-10 shock tunnel is operated by single pulse mode, even the thermodynamic characters are consistent in each operation, the microstructural fluctuation of the flow field from each time is a big issue, especially for the quantitative measurement by dual rotational lines excitation PLIF technique (two-line thermometry). Our preliminary studies found that the deviation of measured flow temperature could be huge and unacceptable when a single beam laser PLIF technique applied to the high-enthalpy reacting flow around blunt body in JF-10 shock tunnel, even while that one 


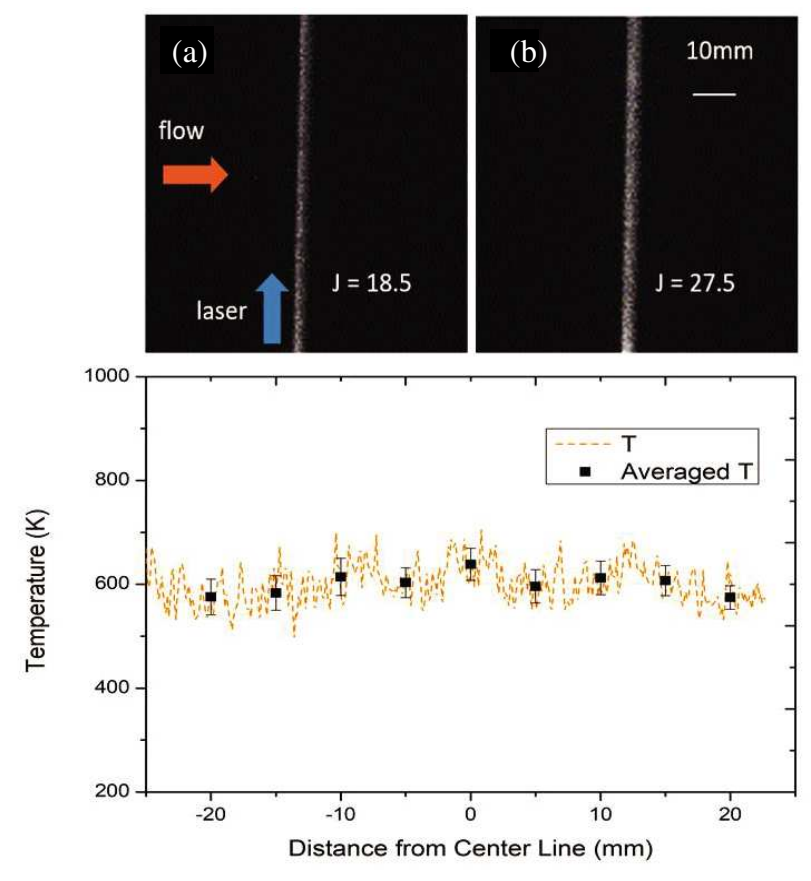

Figure 2: Above: LIF images of free-stream flow in JF-10 shock tunnel with excitation for $\gamma(0,0)$ transitions (a) $R_{21}$ (18.5), (b) $\mathrm{Q}_{1}+\mathrm{P}_{21}$ (27.5) of NO molecules. Below: Distribution of temperature deduced from NOLIF images in JF-10 shock tunnel with free-stream mode.

in free-stream flow is rather acceptable as before mentioned.

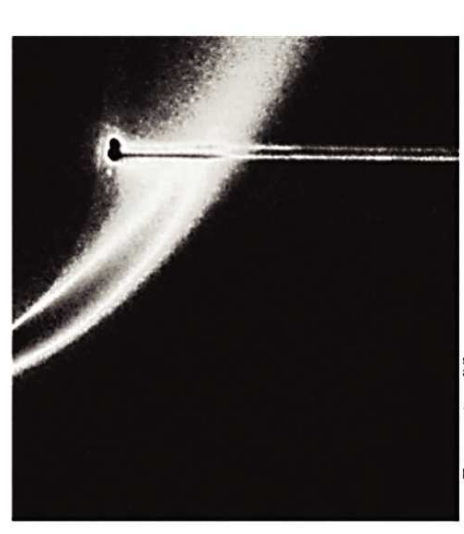

(a)
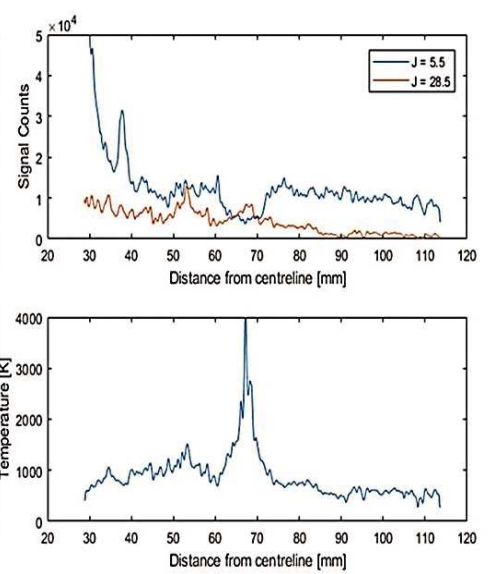

(b)

Figure 3: (a) LIF images of high-enthalpy reacting flow around blunt body in JF-10 shock tunnel with excitation for $\gamma(0,0)$ transitions $\mathrm{Q}_{1}+\mathrm{P}_{21}$ (5.5) and $\mathrm{Q}_{1}+\mathrm{P}_{21}$ (28.5) of $\mathrm{NO}$ molecules; (b) Distributions of NO fluorescence signals along with the laser beam (above); Distribution of temperature in high-enthalpy reacting flow around blunt body in JF-10 shock tunnel deduced from left NO-LIF images (below).

In the case, we established a dual-dye-laser LIF system for flow field investigations and applied it successfully on high-enthalpy reacting flow around blunt body in JF-10 shock tunnel. Experimental results shown that the optimized arrangement is less affected by the fluctuation of the flow field from each facility operation, thus capable of reflect the dramatic temperature change across the shock layer, just as shown in Fig. 3. With precise control of the proper of the laser, and through evaluation of the error of the data, the temperature measurement and numerical simulation in the flow is consistent. 


\subsection{Velocity Measurement on High-enthalpy Reacting Flow around Blunt Body}

At last, ith the same dual-dye-laser LIF apparatus, the velocity of the high-enthalpy reacting flow around blunt body was also measured by molecular tagging velocimetry (MTV) with a long-life fluorescence excitation. The MTV is a "time of flight" technique. The velocity measurement can be easily performed by writing a tag line into the flow and recording its displacements after multiple time delays with no need for the complex calibrations or corrections as in the Doppler shift method.

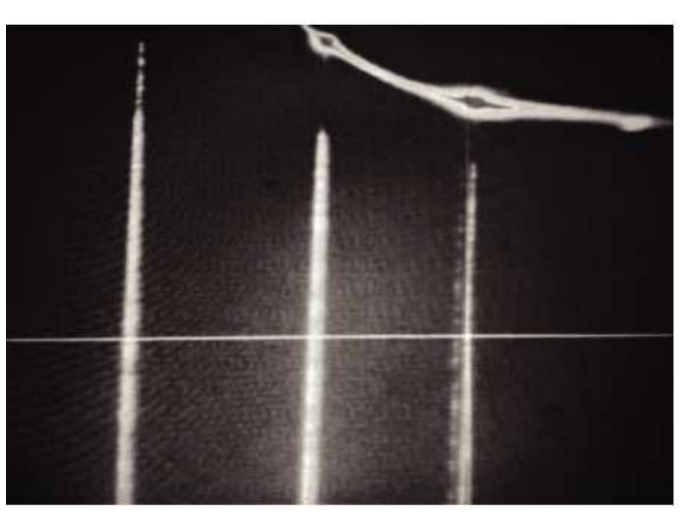

(a)

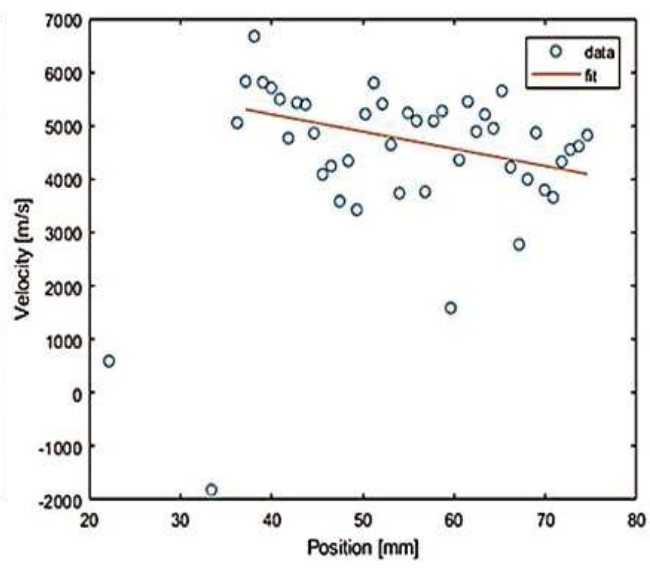

(b)

Figure 4: (a) a series of fluorescence lines with different delays on high-enthalpy reacting flow around blunt body in JF-10 shock tunnel with excitation for $\gamma(0,0)$ transitions Q1+P21 (38.5) of NO molecules; (b) Distribution of velocity in high-enthalpy reacting flow around blunt body in JF-10 shock tunnel by MTV.

Before imaging the tagged region for velocity measurements, a high precision ruler was imaged and number of pixels per $\mathrm{mm}$ in both $x$ - and $y$-directions of the camera was determined in order to calibrate the image plane of the ICCD camera for conversion from pixel number to distance ( $1 \mathrm{~mm} \sim 9.8$ pixel in the present experiment). The acquired images were corrected for pixel nonuniformity, Then the effects of dark charge accumulation and natural arc discharges emissions by a pixel-by-pixel subtraction of an image without excited laser. In the same way, the slope of laser sheet to gas flow was also corrected by subtraction of an image which acquired with zero delay to the laser pulse.

In the present velocity measurement, the excitation lasers were both tuned at the $\gamma(0,0)$ transitions $\mathrm{Q}_{1}+\mathrm{P}_{21}$ (38.5) of $\mathrm{NO}$ molecules, where the fluorescence signal is stronger and can be maintained more longer than other transitions. It is believed that the signal probably not only came from the NO molecules duo to the spectra overlapping of Fe atoms, $\mathrm{NO}$ and $\mathrm{O}_{2}$ molecules. One of the laser beams in dual-dye-laser LIF apparatus was used as "standard line" in delay t0, the other one was splitted to three beams with different delays with "standard line" by "delay line" setup. Then a series of fluorescence lines with different delays were recorded and displayed in Fig. 4(a). Comparing with "standard line" location, the displacements of those fluorescence lines could be obtained. Combined with the measured conversion from pixel number to distance above, the velocities of gases flow were calculated based on the displacements of the tagged region with different delay times $(v=\delta d / \delta t)$, as demonstrated in right part of Fig. 4.

\section{CONCLUSIONS}

In present work, we established a dual-dye-laser Laser-induced Fluorescence system for highenthalpy reacting flow investigations in a jumbo $\mathrm{H}_{2} / \mathrm{O}_{2}$-detonation-driven shock tunnel (Model: JF-10, Total Enthalpy: 16.1 MJ/kg, Static Pressure: 105 Pa) where the effective concentration of NO for PLIF detecting were predicted about $10^{11} \mathrm{~cm}^{-3}$ which is far lower than the detection limit of PLIF ( $\sim 10^{15} \mathrm{~cm}^{-3}$, according to reference [13]). With an optimized optical arrangement and the dual-dye-laser setup, the signal to noise ratio of NO fluorescence in JF10 shock tunnel was notably improved and single-shot measurements were achieved. As a result, the temperature distributions of free-stream and the flow field around blunt body with shock layer were measured, while the velocity of the flow was also obtained by molecular tagging velocimetry (MTV) with a long-life 
fluorescence excitation by the dual-dye-laser LIF apparatus.

\section{ACKNOWLEDGMENT}

The work is partially supported by National Science Foundation of China (Grant No.11672359).

\section{REFERENCES}

1. Hanson, R. K., J. M. Seitzman, and P. H. Paul, "Planar laser-fluorescence imaging of combustion gases," Appl. Phys. B, Vol. 50, 441-454, 1990.

2. Dyer, M. J. and D. R. Crosley, "Two-dimensional imaging of $\mathrm{OH}$ laser-induced fluorescence in a flame," Opt. Lett., Vol. 7, 382-384, 1992.

3. Zimmermann, M., "Resonant doppler velocimeter," Ph.D. Thesis, Princeton University, Trenton, 1980.

4. Wehrmeyer, J. A., L. A. Ribarov, D. A. Oguss, et al., "Flame flow tagging velocimetry with 193-nm $\mathrm{H}_{2} \mathrm{O}$ photodissociation," Appl. Opt., Vol. 38, 6912-6917, 1999.

5. Wollenhaupt, M., M. Rosenhauer, T. Müller, et al., "NO laser-induced fluorescence studies for the application of single-shot two-line thermometry to HEG," 21st International Symposium on Shock Waves, 8360, Great Keppel Island, Australia, July 20-25, 1997.

6. Bessler, W. G., C. Schulz, T. Lee, J. B. Jeffries, and R. K. Hanson, "Strategies for laserinduced fluorescence detection of nitric oxide in high-pressure flames. III. Comparison of A-X excitation schemes," Appl. Opt., Vol. 42, 4922-4936, 2003.

7. Hildenbrand, F., C. Schulz, J. Wolfrum, F. Keller, and E. Wagner, "Laser diagnostic analysis of no formation in a direct injection diesel engine with pump-line-nozzle and common rail injection systems," Proc. Combust. Inst., Vol. 28, 1137-1144, 2000.

8. Ress, J. M., G. Laufer, and R. H. Krauss, "Laser ion time-of-flight velocity measurements using N2+ tracers," AIAA Journal, Vol. 33, No. 2, 296-301, 1995.

9. Ribarov, L. A., J. A. Wehrmeyer, F. Batliwala, R. W. Pitz, and P. A. DeBarber, "Ozone tagging velocimetry using narrowband excimer lasers," AIAA Journal, Vol. 37, No. 6, 708$714,1999$.

10. Ribarov, L. A., J. A. Wehrmeyer, R. W. Pitz, and R. A. Yetter, "Hydroxyl tagging velocimetry (HTV) in experimental airflows," Applied Physics B (Lasers and Optics), Vol. 74, No. 2, 175183, 2002.

11. Palma, P. C., "Laser-induced fluorescence imaging in free-piston shock tunnels," PhD Thesis, 1999.

12. Zeng, H., X. Yu, F. Li, et al., "Nitric oxide concentration and temperature measurement for shock tunnel free stream using mid-infrared absorption spectroscopy," J. Exper. Fluid. Mech., Vol. 29, 79-83, 2015.

13. Mori, H., T. Ishida, S. Hayashi, Y. Aoki, and T. Niimi, "A study on REMPI as a measurement technique for highly rarefied gas flows JSME," International Journal Series B, Vol. 43, No. 3, 400-406, 2000. 